\title{
Antioxidants in Dentistry: Review of Literature
}

\section{Aksakalli $\mathrm{S}^{*}$}

Faculty of Dentistry, Department of Orthodontics, Bezmialem Vakif University, Istanbul, Turkey

\begin{abstract}
An antioxidant is molecule that can inhibit oxidations and free radicals' harmful activities. There are many types of antioxidant products in the market and they can be produced from several sources including minerals, vitamins or food and herbal supplements. In developed countries, consumption of antioxidants has become widespread. As in every medical field, the usage of antioxidants is becoming more frequent. Antioxidants can help adjunct treating the progress of oral problems such as periodontitis or gingivitis. The properties of antioxidants have been studied to understand their mechanisms, to clarify their activities and the increasing evidences are raising hopes about antioxidants for human health.
\end{abstract}

Keywords: Antioxidant; Gingivitis; Low-density lipoprotein

\section{Introduction}

An antioxidant is molecule that can inhibit oxidation of other molecules. Oxidation of molecules produces free radicals. These free radicals can cause harmful chain reactions that are responsible for cell damage or cell death, which can be responsible for carcinogenesis or low-density lipoprotein (LDL) oxidations in cardiovascular diseases. Antioxidants neutralize these radicals by donating their electrons, ending the electron taking reaction. Oxidative stress is a characteristic feature in many diseases. The majority of free radicals that damage biological mechanisms are oxygen-free radicals and these are known as 'reactive oxygen species' (ROS). ROS can be formed during UV light irradiation, by $\mathrm{X}$ rays, gamma rays produced during metal catalyzed reactions are present in the atmosphere as pollutants are produced by neutrophils and macrophages during inflammation and are by products of mitocondrşal catalyzed electron transport systems and various other mechanisms [1]. The disharmony between ROS production and antioxidant numbers to scavenge ROS will increase oxidative stress. ROS are oxygen derived small molecules produced as intermediates in the redox reactions, such as ozone, superoxides, singlet oxygen and hydrogen peroxide. So, ROS play important roles in cellular signaling processes and trigger diseases [2].

Antioxidants are capable of deactivating free radicals before they attack human cells. Humans have generated highly complex antioxidant systems (enzymic or nonenzymic), which work synergistically and in combination with each other to prevent cells or organs against free radicals. The antioxidants can be endogenous or exogenous eg, as a part of diet or dietary supplements [3].

An antioxidant assumed to be effective must contain some properties: a- Enzymatic antioxidants that works by catalyzing the oxidation of different molecules, $b$ - The chain breaking action, $c$-The substances including thiol groups that acts largely sequestering transition metallic ions and damages Fenton reactions [4,5]. By using antioxidants, harmful chain reactions can be annihilated or stopped. Antioxidants can act by being oxidized themselves and they can be divided into sub categories such as ascorbic acids, polyphenols according to their type. They can be divided into two major categories, depending on whether they are soluble in water or in lipids. Water soluble antioxidants react with oxidants in the cell cytosol or blood plasma, while lipid soluble types protect cell membranes from lipid peroxidation. The antioxidants can be synthesized in the body or obtained from the diet [6].

Antioxidants are produced from several sources including minerals, vitamins or food and herbal supplements. These supplements can be acquired in capsule, liquid or tablet forms. In dental field, there are toothpastes, mouth rinses or oral sprays incorporating antioxidant supplements. The majority of supplements include green tea, propolis, grape seed or pine bark extracts $[4,7]$.

The immune system in human-being has been connected to the amount of dietary antioxidants intake [8]. Consumption of antioxidants in developed countries has become widespread [9]. Researchers should be more focused on antioxidants' effects and there should be more researches, randomized controlled trials and final conclusions about their effects-efficiencies or safety.

\section{Periodontology}

Oxidative stress stays at the heart of the periodontal damage that happens from host-pathogen interactions. This stress is a result of excess ROS activity, antioxidant deficiency or activation of redoxsensitive transcription factors and the creation of inflammatory stage. It is known that there are significant relations between oxidant status and periodontal status, the oxidative stress can play important role in the pathology of periodontitis [10].

Antioxidants change the progress of oral problems such as periodontitis, gingivitis by compromising antioxidant capacity of crevicular fluid and plasma. One of the conditioning factors for gingivitis is ascorbic acid deficiency. So, antioxidant support are preferred against struggling periodontal diseases [11,12]. Plant oils and green, leafy vegetables can break free radical chain reactions thus may contribute in reducing periodontal inflammation. Flavanoids acquired from antioxidants can possess anti-inflammatory properties that reduce inflammatory molecule expressions in immune system warriors such as monocytes within the gingival connective tissues [4]. In addition, the cranberry fraction could prevent biofilm formation by Phorphyromonas Gingivalis that is a major pathogen of chronic periodontitis [13]. Carvalho et al. [14] stated that Vitamin E has a potential to reduce oxidative damage in experimental periodontitis but does not prevent alveolar bone loss and could cause anxiety.

\section{Restorative Dentistry}

It was reported that green tea's epigallocatechin-3-gallate molecule

*Corresponding author: Aksakalli S, DDS, PhD, Faculty of Dentistry, Department of Orthodontics, Bezmialem Vakif University, Istanbul, Turkey, Tel: 905333548685 ; E-mail: sertacaksakal@gmail.com

Received November 29, 2013; Accepted December 17, 2013; Published December 19, 2013

Citation: Aksakalli S (2013) Antioxidants in Dentistry: Review of Literature Dentistry 4: 181. doi:10.4172/2161-1122.1000181

Copyright: (c 2013 Aksakalli S. This is an open-access article distributed under the terms of the Creative Commons Attribution License, which permits unrestricted use, distribution, and reproduction in any medium, provided the original author and source are credited. 
had scavenging effect on dental caries prevention. Cranberries, especially their Type A oligomers, were capable of having antibacterial activity against Streptococcus Mutans and stopping dental caries [15]. In the restorative treatment of caries, to increase bond strength of composites, grape seed or pine bark extract solutions can be performed, especially to raise decreased bond strength values for restorative treatments after bleaching $[16,17]$.

\section{Orthodontics}

In orthodontics, similar agents can be used to increase bond strength of brackets $[17,18]$. In bracket bonding, to increase bond strength values ascorbic acid solutions that were hard to prepare were used. In a recent study, it was reported that the usage of pine bark extract solution could be used instead of ascorbic acid solution [18]. For maxillary expansion, there are several studies researching the effect of antioxidant agents on bone formation or maturation. Uysal et al. researched the effect of Vitamin $\mathrm{C}$ and resveratrol in the expanded premaxillary suture. They concluded that the effect of antioxidants on bone formation was statistically significant $[19,20]$. Similarly, Altan et al. revealed that systemic propolis usage stimulates bone formation in the expanded suture area [21].

\section{Oral-Maxillofacial Surgery}

According to Ohnishi et al. [22] reactive oxygen such as hydrogen peroxide, are responsible for the alveolar bone loss that is accompanied by decreased endothelial nitric oxide synthase expression in mice and they stated that the generation of oxidative stress is an underlying systemic condition that enhances alveolar bone loss. Peri-implantitisis initiated by gram negative, anaerobic bacteria that accomodates in the subgingival area. Treating peri-implantitis involves antioxidant supplemention [4]. Sheresta et al. stated that grape seed extract has positive effect on treating peri-implantitis [23]. For bone healing and bone formation, it was reported that caffeic acid phenethyl ester which can be found in propolis have significantly improved bone healing in rat models [24].

\section{Oral Cancer}

Antioxidants show preventive and therapeutic potential in many stages of oral carcinogenesis. Researchers have recently stated there is inhibition of oral cancer phenotypes after antioxidant intakes. The administrations of proanthocyanidins that can be found in flavonoid structures of antioxidants have an ability to reduce cell growth and proliferation of oral carcinomas $[25,26]$. Dietary antioxidants can protect the lipids and other membrane molecules against oxidative damage by intercepting oxidants before they try to destroy the tissues [27].

\section{Question Marks about Antioxidants}

Although antioxidants have an important role in oral-dental treatments, there are some issues need to be studied more. Firstly, the effective amount of antioxidants has not been clearly investigated and varies among populations or species studied. Overdoses or high doses of Vitamin E and Vitamin A can cause health risks such as toxic and teratogenic effects [27]. Drug interactions are other problems about using antioxidants. Simply if any drug enhances free radical activity, the usage of antioxidants will neutralise this effect. In literature, some researches related to antioxidants have a standardization problem. There are lack of protocols for quality control and quality parameters in standardizing antioxidants with respect to potency and safety [4]. Moreover, most of the studies are in vitro or animal studies and there are few clinical studies.
For the selection of antioxidant treatments or their support, a clinician should answer some questions. Are there enough convincing studies in literature for your case? Do we clearly know the adverse effects of the antioxidant we choose? Has oxidative damage been implicated in the illness' pathophysiology? In tissue level, do we know the place where the oxidative damage takes place? Will our antioxidant get to that area?

\section{Conclusion}

In conclusion, the properties of most of the antioxidant have been studied to understand their mechanisms and to clarify their activities. Future researches should continue with the aim of investigating the antioxidant biocompability and understanding their pathways on human health. Some antioxidants have more anti-inflammatory activity whereas some have more anti-tumor properties. So, some researchers have suggested using combined antioxidant supplements thus the supplements will provide higher protection against free radicals [28]. Although there are some debates about research methods, protocols or proper doses, the increasing evidences are raising hopes about antioxidants for human health.

\section{References}

1. Cadenas E (1989) Biochemistry of oxygen toxicity. Annu Rev Biochem 58: 79110.

2. Chen S, Meng XF, Zhang C (2013) Role of NADPH Oxidase-Mediated Reactive Oxygen Species in Podocyte Injury. Biomed Res Int 2013: 839761.

3. Rahman K (2007) Studies on free radicals, antioxidants, and co-factors. Clin Interv Aging 2: 219-236.

4. Carnelio S, Khan SA, Rodrigues G (2008) Definite, probable or dubious: antioxidants trilogy in clinical dentistry. Br Dent J 204: 29-32.

5. Chapple IL, Brock GR, Milward MR, Ling N, Matthews JB (2007) Compromised GCF total antioxidant capacity in periodontitis: cause or effect? J Clin Periodontol 34: 103-110.

6. Sies H (1997) Oxidative stress: oxidants and antioxidants. Exp Physiol 82: 291 295.

7. Abebe W (2003) An overview of herbal supplement utilization with particular emphasis on possible interactions with dental drugs and oral manifestations. J Dent Hyg 77: 37-46.

8. Moskaug JO, Carlsen H, Myhrstad MC, Blomhoff R (2005) Polyphenols and glutathione synthesis regulation. Am J Clin Nutr 81: 277S-283S.

9. Millen AE, Dodd KW, Subar AF (2004) Use of vitamin, mineral, nonvitamin and nonmineral supplements in the United States: The 1987, 1992, and 2000 National Health Interview Survey results. J Am Diet Assoc 104: 942-950.

10. Dahiya P, Kamal R, Gupta R, Bhardwaj R, Chaudhary K, et al. (2013) Reactive oxygen species in periodontitis. J Indian Soc Periodontol 17: 411-416.

11. Maxwell SR (1995) Prospects for the use of antioxidant therapies. Drugs 49 345-361.

12. Nakamoto T, McCroskey M, Mallek HM (1984) The role of ascorbic acid deficiency in human gingivitis--a new hypothesis. J Theor Biol 108: 163-171.

13. Labrecque J, Bodet C, Chandad F, Grenier D (2006) Effects of a high-molecularweight cranberry fraction on growth, biofilm formation and adherence of Porphyromonas gingivalis. J Antimicrob Chemother 58: 439-443.

14. Carvalho Rde S, de Souza CM, Neves JC, Holanda-Pinto SA, Pinto LM, et al. (2013) Vitamin $E$ does not prevent bone loss and induced anxiety in rats with ligature-induced periodontitis. Arch Oral Biol 58: 50-58.

15. Schmidt MA, Riley LW, Benz I (2003) Sweet new world: glycoproteins in bacterial pathogens. Trends Microbiol 11: 554-561.

16. Berger SB, De Souza Carreira RP, Guiraldo RD, Lopes MB, Pavan S, et al. (2013) Can green tea be used to reverse compromised bond strength after bleaching? Eur J Oral Sci 121: 377-381.

17. Vidhya S, Srinivasulu S, Sujatha M, Mahalaxmi S (2011) Effect of grape seed extract on the bond strength of bleached enamel. Oper Dent 36: 433-438. 
18. Aksakalli S, lleri Z, Karacam N (2013) Effect of pine bark extract on bond strength of brackets bonded to bleached human tooth enamel. Acta Odontol Scand 71: 1555-1559.

19. Uysal T, Gorgulu S, Yagci A, Karslioglu Y, Gunhan O, et al. (2011) Effect of resveratrol on bone formation in the expanded inter-premaxillary suture: early bone changes. Orthod Craniofac Res 14: 80-87.

20. Uysal T, Amasyali M, Olmez H, Enhos S, Karslioglu Y, et al. (2011) Effect of vitamin $\mathrm{C}$ on bone formation in the expanded inter-premaxillary suture. Early bone changes. J Orofac Orthop 72: 290-300.

21. Altan BA, Kara IM, Nalcaci R, Ozan F, Erdogan SM, et al. (2013) Systemic propolis stimulates new bone formation at the expanded suture: a histomorphometric study. Angle Orthod 83: 286-291.

22. Ohnishi T, Bandow K, Kakimoto K, Machigashira M, Matsuyama T, et al. (2009) Oxidative stress causes alveolar bone loss in metabolic syndrome model mice with type 2 diabetes. J Periodontal Res 44: 43-51.

23. Shrestha B, Theerathavaj ML, Thaweboon S, Thaweboon B (2012) In vitro antimicrobial effects of grape seed extract on peri-implantitis microflora in craniofacial implants. Asian Pac J Trop Biomed 2: 822-825.

24. Ucan MC, Koparal M, Agacayak S, Gunay A, Ozgoz M, et al. (2013) Influence of caffeic acid phenethyl ester on bone healing in a rat model. J Int Med Res 41: 1648-1654.

25. King M, Chatelain K, Farris D, Jensen D, Pickup J, et al. (2007) Oral squamous cell carcinoma proliferative phenotype is modulated by proanthocyanidins: a potential prevention and treatment alternative for oral cancer. BMC Complement Altern Med 7: 22.

26. Shirataki Y, Kawase M, Saito S, Kurihara T, Tanaka W, et al. (2000) Selective cytotoxic activity of grape peel and seed extracts against oral tumor cell lines. Anticancer Res 20: 423-426.

27. Skibsted LH, Dragsted LO, Dyerberg J, Hansen HS, Kiens B, et al. (2006) [Antioxidants and health]. Ugeskr Laeger 168: 2787-2789.

28. San Miguel SM, Opperman LA, Allen EP, Svoboda KK (2011) Use of antioxidants in oral healthcare. Compend Contin Educ Dent 32: E156-159. 